\title{
INTERVENÇÃO FONOAUDIOLÓGICA PARA CONSULTORES EM UM SERVIÇO DE TELEATENDIMENTO: BEM-ESTAR VOCAL
}

\section{Vocal intervention for telemarketing service consultants: vocal well-being}

\author{
Taís de Campos Moreira ${ }^{(1)}$, Mauriceia Cassol (2), Samara Regina Fávero ${ }^{(3)}$, \\ Lyssandra Borba Oliveira ${ }^{(4)}$, Cristiane da Silva Longaray ${ }^{(5)}$, Midiany de Oliveira Soares ${ }^{(6)}$, \\ Michelle Dourado Ramos ${ }^{(7)}$, Ísis Rauber Guilherme ${ }^{(8)}$, Deisi Vidor ${ }^{(9)}$, \\ Maristela Ferigolo ${ }^{(10)}$, Helena Maria Tannhauser Barros ${ }^{(11)}$
}

\section{RESUMO}

Objetivo: verificar efeitos de um programa de bem-estar vocal aos consultores de um serviço de teleatendimento para a saúde. Métodos: participaram do estudo 27 consultores do Call Center VivaVoz que foram avaliados pré e pós-treinamento de voz por meio de análise perceptivo-auditiva da qualidade vocal, articulação, velocidade de fala, ressonância, intensidade e freqüência, por meio da emissão de vogais sustentadas e de fala encadeada com contagem de números de 1 à 20 e a emissão dos dias da semana. O registro de voz foi realizado em sistema digital e a escala japonesa GRBAS-I como instrumento de análise. Também foram realizadas a auto-avaliação vocal e o levantamento dos sintomas vocais. Atividades de voz foram divididas em cinco oficinas sobre saúde vocal, técnicas de aquecimento e desaquecimento, articulação e respiração. Análises descritivas e bivariadas foram realizadas, utilizando Teste T para amostras pareadas e Teste de McNemar. Resultados: os resultados pré-intervenção são de que $50 \%$ das mulheres e $33 \%$ dos homens consultores apresentaram alterações em relação à respiração, $50 \%$ das consultoras com alterações na qualidade vocal e $33 \%$ dos homens dificuldades em relação à articulação. Após as oficinas, os resultados mostraram melhora da qualidade vocal, do padrão articulatório e da fluência de fala, bem como da satisfação vocal nos relatos dos consultores. Conclusão: observaram-se mudanças positivas na qualidade do atendimento à população, além da ampliação do conhecimento dos teleatendentes em relação ao uso correto da voz e manutenção do bem-estar vocal.

DESCRITORES: Voz; Consultores; Treinamento da Voz

(1) Fonoaudióloga; Supervisora do VIVAVOZ da Universidade Federal de Ciências da Saúde de Porto Alegre, UFCSPA, Porto Alegre, RS; Mestre em Ciências Médicas pela Universidade Federal de Ciências da Saúde de Porto Alegre; Doutoranda em Ciências da Saúde na Universidade Federal de Ciências da Saúde de Porto Alegre, Porto Alegre, Rio Grande do Sul.

(2) Fonoaudióloga; Diretora do Curso de Fonoaudiologia da Universidade Federal de Ciências da Saúde de Porto Alegre, UFCSPA, Porto Alegre, RS; Doutora em Medicina e Ciências da Saúde pela Pontifícia Universidade Católica do Rio Grande do Sul, Porto Alegre, Rio Grande do Sul.

(3) Aluna do curso de Graduação em Fonoaudiologia da Universidade Federal de Ciências da Saúde de Porto Alegre, UFCSPA, Porto Alegre, RS.

(4) Aluna do curso de Graduação em Fonoaudiologia da Universidade Federal de Ciências da Saúde de Porto Alegre, UFCSPA, Porto Alegre, RS.

(5) Aluna do curso de Graduação em Fonoaudiologia da Universidade Federal de Ciências da Saúde de Porto Alegre, UFCSPA, Porto Alegre, RS.

(6) Aluna do curso de Graduação em Fonoaudiologia da Universidade Federal de Ciências da Saúde de Porto Alegre, UFCSPA, Porto Alegre, RS.
(7) Aluna do curso de Graduação em Fonoaudiologia da Universidade Federal de Ciências da Saúde de Porto Alegre, UFCSPA, Porto Alegre, RS.

(8) Aluna do curso de Graduação em Fonoaudiologia da Universidade Federal de Ciências da Saúde de Porto Alegre, UFCSPA, Porto Alegre, RS.

(9) Fonoaudióloga; Professora do curso de Fonoaudiologia da Universidade Federal de Ciências da Saúde de Porto Alegre, UFCSPA, Porto Alegre, RS; Doutora em Linguística e Letras pela Pontifícia Universidade Católica do Rio Grande do Sul, Porto Alegre, Rio Grande do Sul.

(10) Farmacêutica; Coordenadora do VIVAVOZ da Universidade Federal de Ciências da Saúde de Porto Alegre, UFCSPA, Porto Alegre, RS; Doutora em Ciências Médicas pela.Universidade Federal do Rio Grande do Sul, Porto Alegre, Rio Grande do Sul.

(11) Médica; Coordenadora do VIVAVOZ da Universidade Federal de Ciências da Saúde de Porto Alegre, UFCSPA, Porto Alegre, RS; Pós-doutora em Neuropsicofarmacologia pela Tufts University, Boston, Massachusetts, EUA.

Conflito de interesses: inexistente 


\section{INTRODUÇÃO}

A voz tem sido muito utilizada como instrumento de trabalho e ascensão profissional em nossa sociedade. Com o advir dos novos tempos, um dos serviços mais modernos e que tendem a crescer ainda mais expressivamente entre nós é o de teleatendimento, no qual a voz é instrumento para o mais variado leque de objetivos, desde os comerciais até os assistenciais.

O crescimento constante desta modalidade de trabalho trouxe a necessidade do aperfeiçoamento do operador de teleatendimento, em função do importante papel que este desempenha na comunicação entre a empresa e os clientes, e exigindo deste profissional um autocontrole emocional significativo, para tratar com demandas pessoais específicas e, geralmente, difíceis ${ }^{1}$.

Nesse contexto a voz é constantemente utilizada, e, por isso, ela deve ser clara e precisa ${ }^{2,3}$. Quando produzida em intensidade e projeção adequada, proporciona um bom desempenho vocal para estes profissionais ${ }^{4}$. Além disso, é fundamental que os operadores apresentem requisitos, com boa fluência verbal, vocabulário amplo, capacidade de ouvir, rapidez de raciocínio, iniciativa, equilíbrio emocional, paciência e cordialidade.

Apesar disso, os trabalhadores de call center geralmente não possuem orientação para melhor utilizarem sua voz, realizando vários usos vocais incorretos. Com isso, há um aumento na probabilidade do desenvolvimento de disfonias do tipo funcional, que por sua vez poderão gerar futuras lesões secundárias por trauma da mucosa das pregas vocais ${ }^{2}$. Os teleoperadores frequentemente apresentam queixas de ressecamento, cansaço para falar, esforço, pigarro, tosse, perda e falhas na voz, rouquidão, ardência e corpo estranho na garganta. Muitos desses sintomas estão relacionados a comportamentos de mau uso e abuso da voz. Também podem estar relacionados a fatores ambientais desfavoráveis, tais como ar condicionado, ruído ambiental excessivo e mobiliário impróprio, ou a fatores ligados ao comportamento do próprio indivíduo, como tensão muscular, falta de suporte respiratório, postura alterada, consumo de bebidas geladas, alcoolismo, tabagismo, pouca ingestão de água e alimentação inadequada, entre outros ${ }^{5,6}$.

Por ser a voz o principal instrumento de trabalho nas empresas de teleatendimento, faz-se necessária a presença do fonoaudiólogo. Uma vez reconhecidos os principais fatores de risco, este profissional pode atuar para sanar, orientar e intervir no ambiente de trabalho para melhoria do conforto e funcionalidade, e minimizar os fatores que poderão comprometer a saúde dos teleoperadores e a qualidade vocal ${ }^{7}$. O fonoaudiólogo também pode participar na seleção bem como no treinamento dos profissionais, colaborar com a prevenção de futuros problemas de comunicação, favorecer melhorias na qualidade do atendimento telefônico e implantar programas de bem-estar vocal $2,8,9$.

Desde 2005, uma central telefônica aberta à população de todo país vem sendo usada para informações sobre saúde. Esta central é o serviço VIVAVOZ, concebido pela Secretaria Nacional de Políticas sobre Drogas (SENAD) e a Universidade Federal de Ciências da Saúde de Porto Alegre (UFCSPA). O VIVAVOZ presta aconselhamento telefônico reativo, gratuito, anônimo, personalizado e especializado em oferecer informações e orientações sobre as substâncias psicoativas com intervenção breve motivacional ${ }^{10,11}$. A partir de 2007 , com a implantação do Curso de Fonoaudiologia na UFCSPA, um grupo de alunos, coordenados por fonoaudiólogos, tem realizado avaliações e orientações aos teleoperadores chamados de consultores, desta central telefônica, por intermédio de projetos de extensão.

Considerando-se a importância do uso da voz em serviços telefônicos, não só relativo ao bemestar dos profissionais que ali trabalham, mas também na qualidade e efetividade do atendimento prestado, justifica-se a necessidade do fonoaudiólogo na avaliação e prevenção de distúrbios vocais nesta população.Cuidar da voz de teleoperadores significa mais que um treinamento, se levarmos em conta o impacto emocional de uma alteração vocal limitadora da profissão. Assim, o principal objetivo deste artigo é investigar os efeitos de um programa de bem-estar vocal aos consultores do call center VIVAVOZ.

\section{MÉTODOS}

A presente pesquisa trata-se de um estudo quase-experimental de intervenção não-controlado $^{12}$, no qual foram comparados os dados obtidos por meio da avaliação vocal pré e pós-oficinas de orientação realizadas semanalmente com os teleoperadores do serviço VIVAVOZ, pelo período de cinco semanas. O VIVAVOZ é uma central telefônica com dez estações de atendimento com ambiente de trabalho planejado, com controle de temperatura e iluminação adequada para um bom desempenho das atividades.

Participaram do programa de bem-estar vocal, realizado em duas edições, uma no ano de 2007 e a outra no de 2008, 46 consultores do call-center VIVAVOZ entre homens e mulheres, com idade entre 18 e 40 anos, que trabalhavam de 4 a 6 
horas/diárias. Da amostra inicial, foram excluídos 19 participantes, pois os mesmos não concluíram o estágio no call center e, portanto, não puderam ser reavaliados. Assim, os resultados desta pesquisa se referem a 27 indivíduos que passaram por todas as etapas: avaliação, oficinas e reavaliação.

A coleta de dados foi realizada por intermédio de uma entrevista individual com todos os teleatendentes call center e incluía dados de identificação dos consultores, bem como aspectos relevantes para o seu trabalho e relatos referentes a sua participação nas oficinas de bem-estar vocal. Para a avaliação e reavaliação utilizaram-se os protocolos elaborados por Bacha ${ }^{13}$ e adaptados por Moreira ${ }^{14}$. Foram eles: questionário sobre hábitos vocais, breve entrevista de identificação, avaliação do perfil fonoaudiológico do operador de telemarketing e realização da avaliação perceptivo-auditiva utilizando a escala GRBAS-I 15,16. Além disso, foi realizada avaliação qualitativa dos relatos dos consultores na gravação para avaliação da percepção de suas vozes. Os protocolos utilizados foram os mesmos para pré e pós intervenção.

O primeiro instrumento aplicado foi o questionário para levantamento dos dados de identificação e profissionais. Posteriormente aplicou-se questões sobre hábitos vocais, no qual os consultores foram instruídos a assinalar apenas os hábitos que faziam uso. Na sequência utilizou-se o protocolo de avaliação do perfil fonoaudiológico do operador de telemarketing, em que se analisou a adequação ou não de parâmetros como, articulação, tipo respiratório, ressonância, intensidade vocal, velocidade de fala, tensão e qualidade vocal. Em relação a avaliação da respiração foram verificados os tempos máximos de fonação a partir da emissão das vogais sustentadas /a/, /i/, /u/, /e/, /o/, /u/, e dos fonemas fricativos surdo e sonoro /s/ e/z/, utilizando o cronômetro para marcar o tempo.O tipo respiratório foi avaliado pelas medidas dos diâmetros nas alturas alto, médio e baixo durante a respiração. Ao final da avaliação, os teleoperadores foram orientados a seguir um roteiro de gravação de voz ${ }^{10}, \mathrm{com} 0$ objetivo de avaliar articulação e velocidade de fala por meio da fala encadeada, no qual deveriam falar seus nomes, idade, a data da entrevista, emitir as vogais /a/, /e/, /i/, /o/, /u/,contar os números de 1 a 20, falar os dias da semana, os meses do ano, cantar a música "Parabéns para você", e por fim, fazer um breve comentário sobre a própria voz. A tensão vocal foi analisada durante a emissão dos sons observando a postura de ombros, peitoral e laringe elevada no pescoço.Para avaliação da ressonância da voz foi utilizada a emissão da vogal /i/ sustentada, e para avaliar a qualidade vocal a emissão da vogal sustentada /a/. O registro das vozes foi realizado utilizando-se o gravador digital PANASONIC, modelo RR-US450. Os participantes do estudo foram orientados a permanecer em pé durante a gravação. Na emissão das vogais, deveriam manter uma distância de cinco centímetros da boca até o microfone, e na fala encadeada dez centímetros, para evitar ruídos no registro de gravação da voz. As avaliações foram realizadas em ambiente silente e todos os aspectos avaliados serviram para verificar os efeitos do bem-estar vocal ${ }^{17}$.

Ao término da primeira avaliação, as fonoaudiólogas responsáveis, juntamente com as seis acadêmicas do curso de Fonoaudiologia, realizaram, a partir das gravações, a análise perceptivo-auditiva com o auxílio da escala GRBAS-I. A escala japonesa GRBAS-I é um método simples de avaliação ${ }^{15}$ da percepção da qualidade de voz, sendo amplamente utilizada no Japão e com crescente interesse na Europa e nos Estados Unidos ${ }^{18}$. Avalia o grau global de disfonia $(G)$, pela identificação da contribuição de quatro fatores independentes: rugosidade (R- roughness), soprosidade (B- breathiness), astenia (A- asteny), tensão ( $\mathrm{S}$ - strain), instabilidade (I- instability) ${ }^{15,16}$. Para a identificação do grau de desvio de cada um dos fatores, uma escala de quatro pontos foi utilizada, sendo que "0" significava normal ou ausente, "1" discreto, " 2 " moderado e " 3 " severo ${ }^{13}$.

Após as avaliações, foram realizadas cinco oficinas, nas quais foram abordados diversos temas. Na primeira oficina, foram trabalhados com os consultores aspectos relacionados à atuação do profissional fonoaudiólogo na área de teleatendimento, bem como, foram esclarecidos os procedimentos que seriam realizados nas oficinas subseqüentes e seu impacto no exercício profissional destes indivíduos. Na oficina de Saúde vocal foram abordados os cuidados para o uso adequado da voz, com o auxílio de folders explicativos contendo informações de hábitos vocais negativos e positivos para a saúde vocal.

Na oficina de Produção vocal, foram exibidos vídeos explicativos sobre a anatomofisiologia da respiração e da fonação, bem como realizados alguns exercícios enfatizando a postura respiratória adequada. Ainda foram dadas orientações quanto à postura corporal, tais como alongamento de pescoço e ombros, postura adequada de permanecer sentado durante $o$ atendimento e apoio dos pés. Na quarta oficina, foram trabalhados exercícios articulatórios, trava-línguas, exercícios utilizando uma rolha entre os dentes e sobrearticulação dos sons.

$\mathrm{Na}$ última oficina, de aquecimento e desaquecimento vocal, foi explicada a importância do 
aquecimento vocal para o profissional da voz antes do trabalho, pois o aparelho fonador necessita de condicionamento muscular favorável, e ao final das atividades é necessário relaxar e desaquecer. Para isso foram utilizados exercícios de aquecimento vocal com sons vibrantes, nasais e sons fricativos para desaquecer a voz ${ }^{3}$.

Cada oficina teve duração de 30 minutos e foram ministradas por duas fonoaudiólogas, com o auxílio de seis acadêmicas de fonoaudiologia. Ao término desses procedimentos, foram iniciadas as reavaliações que seguiram os mesmos moldes das avaliações ${ }^{13}$. Foram regravadas as vozes dos teleoperadores e reavaliadas de forma perceptivoauditiva com o auxílio da escala GRBAS-I, com a finalidade de se verificar a eficiência do treinamento vocal na manutenção e promoção do bem-estar e da saúde da voz dos teleoperadores. O tempo entre as avaliações e reavaliações foi em média de quatro meses, sendo que as oficinas ocorreram dentro desse período.

Esse estudo foi previamente aprovado pelo Comitê de Ética em Pesquisa da Instituição (437/07).

A análise descritiva foi utilizada para estimar a distribuição das variáveis, que foram apresentadas em tabelas de frequências absoluta e relativa. Aplicou-se teste de McNemar e teste T de amostras pareadas para comparação das variáveis categóricas para os parâmetros vocais, tempos máximos de fonação e escala GRBAS-I. Foi considerado estatisticamente significante o valor de $P<0,05$.

\section{RESULTADOS}

Foram utilizados dados obtidos a partir das avaliações de 27 consultores, $24(88,8 \%)$ mulheres e $3(11,2 \%)$ homens, ambos com média de idade de 23 anos. O perfil de saúde vocal dos teleoperadores foi apresentado inicialmente a fim de se conhecer a amostra avaliada (Tabela 1).

As comparações do perfil fonoaudiológico das mulheres e dos homens quanto à adequação antes e depois das oficinas e em relação aos parâmetros avaliados estão citadas na Tabela 2. Observaram-se melhorias em todos os parâmetros, nas mulheres, exceto articulação. Nos dados que se referem aos homens, apenas o parâmetro respiração não mostrou melhora (Tabela 2), embora os dados não apresentaram significância estatística.

Nos tempos máximos de fonação (TMF) os valores obtidos para homens e mulheres após as oficinas de voz, não foram estatisticamente significativos, com exceção do TMF da vogal /a/ no grupo das mulheres $(p=0,02)$, (Tabela 3$)$.

Tabela 1 - Perfil de saúde vocal dos consultores $(\mathrm{N}=27)$ antes da intervenção fonoaudiológica conforme o sexo

\begin{tabular}{lcc}
\hline Hábitos & Mulheres $\mathbf{n = 2 7}$ & Homens $\mathbf{n}=\mathbf{3}$ \\
& $\%$ & $\%$ \\
\hline 1-Costumo tomar, no máximo 5 copos de água durante o dia & 56,5 & 0 \\
2-Costumo falar em ambientes secos e empoeirados & 34,8 & 0 \\
3-Costumo falar em ambientes barulhentos & 56,5 & 33,3 \\
4-Estou sempre gritando ao telefone & 17,4 & 0 \\
5-Estou sempre tossindo e pigarreando & 34,8 & 33,3 \\
6-Mesmo quando estou gripado, costumo falar bastante & 56,5 & 33,3 \\
7-Enquanto prático exercícios físicos, também converso & 21,7 & 33,3 \\
8-Não pratico exercícios físicos frequentemente & 65,2 & 66,7 \\
9-Fumo e converso muito em ambientes de fumantes & 21,7 & 0 \\
10-Costumo beber bebidas alcoólicas & 21,7 & 33,3 \\
11-Quando estou no período pré-menstrual, costumo falar bastante. & 26,1 & 0 \\
12-Falo bastante & 65,2 & 66,7 \\
13-Costumo rir alto & 34,8 & 0 \\
14-Discuto com freqüência & 13 & 0 \\
15-Costumo cantar ou participo de corais & 30,4 & 66,7 \\
16-Tenho azia frequente & 13 & 0 \\
\hline
\end{tabular}

Os dados foram apresentados em porcentagem 
Tabela 2 - Comparação do perfil fonoaudiológico das mulheres $(\mathrm{N}=24)$ e dos homens $(\mathrm{N}=3)$ quanto à adequação dos parâmetros vocais antes e depois das oficinas

\begin{tabular}{lcccccc}
\hline \multirow{2}{*}{ Parâmetros Avaliados } & \multicolumn{3}{c}{ Mulheres } & \multicolumn{3}{c}{ Homens } \\
\cline { 2 - 6 } & Antes & Depois & P & Antes & Depois & P \\
\hline Articulação & $87 \%$ & $82,6 \%$ & NS & $33,3 \%$ & $66,7 \%$ & NS \\
Tipo respiratório & $47,8 \%$ & $82,6 \%$ & NS & $100 \%$ & $66,7 \%$ & NS \\
Ressonância & $30,4 \%$ & $69,6 \%$ & NS & $33,3 \%$ & $66,7 \%$ & NS \\
Intensidade & $65,2 \%$ & $82,6 \%$ & NS & $33,3 \%$ & $100 \%$ & NS \\
Frequência & $78,3 \%$ & $87 \%$ & NS & $66,7 \%$ & $100 \%$ & NS \\
Velocidade de fala & $73,9 \%$ & $91,3 \%$ & NS & $66,7 \%$ & $100 \%$ & NS \\
Tensão & $52,2 \%$ & $87 \%$ & NS & 0 & $100 \%$ & NS \\
Qualidade vocal & $47,8 \%$ & $69,6 \%$ & NS & $33,3 \%$ & $100 \%$ & NS \\
\hline
\end{tabular}

Os dados foram apresentados em porcentagem. Realizado Teste de McNemar, foi considerado estatisticamente significante $\mathrm{p}<0,05^{\star}$. NS= P não significativo.

Tabela 3 - Comparação dos resultados de tempos máximos de fonação (TMF) antes e depois das oficinas conforme o sexo

\begin{tabular}{|c|c|c|c|c|c|c|}
\hline \multirow{3}{*}{ TMF vogais/fricativos } & \multicolumn{3}{|c|}{ Mulheres $n=24$} & \multicolumn{3}{|c|}{ Homens $n=3$} \\
\hline & \multicolumn{2}{|c|}{ Média em segundos } & \multirow{2}{*}{$\mathbf{P}$} & \multicolumn{2}{|c|}{ Média em segundos } & \multirow{2}{*}{$\mathbf{P}$} \\
\hline & Antes & Depois & & Antes & Depois & \\
\hline$/ a /$ & 9,8 & 11,8 & $0,02^{*}$ & 9,5 & 8,9 & 0,83 \\
\hline le/ & 10 & 11 & 0,17 & 7,8 & 7,9 & 0,96 \\
\hline /i/ & 9,9 & 11,8 & 0,06 & 10,1 & 8,6 & 0,44 \\
\hline /o/ & 10,2 & 11,2 & 0,19 & 9,6 & 8,4 & 0,22 \\
\hline$/ u /$ & 10,2 & 18,2 & 0,19 & 9,6 & 9,2 & 0,81 \\
\hline /s/ & 13,4 & 14 & 0,30 & 11 & 11,8 & 0,63 \\
\hline$|z|$ & 11,9 & 13 & 0,17 & 8,6 & 11,1 & 0,08 \\
\hline $\mathrm{s} / \mathrm{z}$ & 1,13 & 1,06 & 0,28 & 1,20 & 1,05 & 0,29 \\
\hline
\end{tabular}

Os valores foram apresentados em segundos. Realizado Teste T para amostras pareadas, foi considerado estatisticamente significante $\mathrm{p}<0,05^{*}$.

Quanto aos valores analisados da escala GRBAS-I, antes e depois das oficinas de voz, perceberam-se resultados significativos apenas em relação ao item astenia $(P=0,00)$ indicando uma melhora na qualidade vocal. Em relação ao grau de alteração global da voz, rouquidão, soprosidade, tensão e instabilidade os dados encontrados não foram significativos.

Em relação aos relatos dos consultores sobre a autopercepção de sua voz toda a amostra referiu ter alguma insatisfação na produção vocal.

\section{DISCUSSÃO}

Os atendentes do Call Center VivaVoz apresentaram alta prevalência de hábitos inadequados para boa saúde vocal antes do procedimento da intervenção, incluindo-se falar muito e não praticar exercícios frequentemente. Em um estudo com teleoperadores $^{19}$, a amostra também apresentou usos incorretos da voz, principalmente o hábito de falar em demasia. No presente trabalho verificouse mudanças positivas após aplicação do programa de bem-estar vocal aos consultores do teleatendimento especificamente no comportamento vocal e na dinâmica respiratória, embora sem significância estatística Em trabalho semelhante, com população de professores, verificou-se melhorias na qualidade vocal após a realização de vivências realizadas por meio de oficinas ${ }^{20}$.

O operador de telemarketing, profissional da voz que possui demanda vocal e operacional, é inserido numa realidade de trabalho que envolve metas e atendimentos com tempos a serem seguidos ${ }^{21}$. Essa exigência vocal acarreta o surgimento de 
alterações que comprometem a voz, prejudicam ou mesmo impedem o exercício da atividade ${ }^{22}$. Em função disso, os operadores de call center apresentam prevalência maior de problemas de voz, que estão associados a fatores de risco modificáveis ${ }^{23}$. Assim, se faz necessária a diminuição da demanda vocal e mudanças em determinados fatores, como o tabagismo, o uso excessivo de medicamentos, problemas respiratórios e sedentarismo, entre outros ${ }^{24}$.

A voz é um dos principais recursos e instrumento de trabalho, além de importante forma de expressão, vinculação, comunicação intersubjetiva e importante elo de relação interpessoal ${ }^{6}$. Com os relatos dos consultores foi observada uma elevada insatisfação quanto à autopercepção de suas vozes. Este dado leva a uma importante reflexão, já que os mesmos utilizam a voz que "não gostam" para o exercício de sua atividade ocupacional. Alterações relacionadas à saúde física dos teleoperadores e fatores organizacionais podem ser responsáveis por justificar a presença de sintomas vocais e, conseqüentemente, a insatisfação com a própria $\mathrm{voz}^{25}$.

No estudo realizado com os consultores do VIVAVOZ, foi possível observar após a realização das oficinas melhorias nos padrões do perfil vocal principalmente nas mulheres. Esse dado foi semeIhante ao estudo realizado com operadores de call center, porém com um número maior de participantes ${ }^{26}$, onde realizaram uma avaliação do programa de treinamento de dois dias. Dentre os operadores do sexo feminino, muitos relataram diminuição da fadiga vocal, da sensação de muco, do pigarro ao longo do dia e melhora dos hábitos vocais devido ao treinamento. Ferreira et al. ${ }^{19}$ referem que mesmo um curso de treinamento vocal curto pode contribuir positivamente para a diminuição de sintomas vocais e para o bem-estar das pessoas que trabaIham constantemente com a voz ${ }^{19}$.

No presente estudo constatou-se, após a realização das oficinas, que nas mulheres ocorreu um aumento na porcentagem de adequação de parâmetros como postura vocal, tipo respiratório, ressonância, intensidade, altura, velocidade de fala, grau de tensão e qualidade vocal. Nos homens os mesmos itens tiveram melhorias, com exceção do parâmetro respiração que não foi modificado. Em trabalho que teve como objetivo avaliar a eficácia de um programa fonoaudiológico para formação de locutores ${ }^{27}$, verificou-se também que após a aplicação do programa sobre o uso vocal os parâmetros de qualidade vocal, ressonância, coordenação pneumofonoarticulatória, articulação, ritmo e velocidade de fala melhoraram significativamente. ${ }^{27}$ Este comparativo pode ser observado por meio dos testes descritos no método.A relação $\mathrm{s} / \mathrm{z}$ deve apresentar valores entre 0,8 e 1,2 em indivíduos normais, valores inferiores são indicativos de hiperadução das pregas vocais e valores acima de 1,2 revelam falta de coaptação glótica ${ }^{28}$. Após a intervenção nos consultores do VIVAVOZ, o valor da média em segundos dos tempos máximos de fonação (TMF) das mulheres demonstrou um considerado aumento em todas as vogais testadas. No grupo dos homens esta evolução foi parcial, ou seja, houve aumento do tempo de fonação das vogais, porém sem significância estatística.

Os dados obtidos por meio da avaliação perceptivo-auditiva da escala GRBAS-I foram importantes para investigar a qualidade vocal dos participantes antes e depois da intervenção vocal. Na comparação dos resultados, foi possível observar uma melhora no grau de classificação da escala tanto nas mulheres, quanto nos homens. Esta melhora decorreu do treinamento realizado que enfatizou o uso de técnicas com a emissão de sons facilitadores fricativos, nasais e vibrantes, com modulações de intensidade e freqüência que interferem diretamente no movimento vibratório das pregas vocais e estimulam o uso pleno das cavidades de ressonância ${ }^{28}$.

Após a intervenção fonoaudiológica, evidenciouse um aumento da percepção dos teleoperadores, quanto às suas vozes, devido ao treinamento auditivo com a gravação de áudio e vídeo realizadas nas oficinas e após exibidas ao grupo para estimular a auto-avaliação vocal. Segundo autores ${ }^{25,29}$, a conscientização da voz é um instrumento importante para a comunicação bem-sucedida no trabalho.

A atuação fonoaudiológica em centrais de telemarketing teve início há aproximadamente 12 anos, porém, eram realizados apenas trabalhos esporádicos $\mathrm{e}$ isolados ${ }^{18}$. A constante presença do fonoaudiólogo em telemarketing se faz necessária justamente porque a voz é o instrumento de trabalho e,consequentemente foco de preocupação destas empresas $^{30}$. Portanto, este profissional pode participar desde a criação da central, na seleção dos teleoperadores, no treinamento, monitoramento da equipe, no aperfeiçoamento da comunicação, bem como na prevenção de doenças ocupacionais ${ }^{30}$. Atualmente, para atuação em um call centeré necessário focar não apenas a voz, mas também a fala, a linguagem e a audição auxiliando na prevenção de alterações vocais, na promoção de saúde e no aprimoramento da habilidade comunicativa favorecendo assim a qualidade do atendi-mento ${ }^{9,30}$. Esses parâmetros, nos consultores do VivaVoz, serão avaliados em estudos futuros. Em um estudo realizado com o objetivo de caracterizar o perfil vocal do operador de telemarketing de uma empresa ${ }^{31}$, os sujeitos atribuíram melhora da voz ao uso de pastiIhas e balas, repouso vocal e ingestão de água e além de, relatarem piora vocal com o consumo de 
líquidos ou alimentos muito gelados e uso intensivo de voz. Os operadores ainda reforçaram a necessidade de melhora do ambiente físico de trabalho e adequação da carga horária, assim como treinamento e assessorias vocal e psicológica ${ }^{31}$. Autores afirmam que o treinamento vocal com aquecimento afeta variados aspectos da produção da voz profissional, tanto para homens como para mulheres, sendo influenciado por fatores ambientais e ergonômicos, em especial nos indivíduos que relatam mais fadiga, menos motivação, mais desconforto e outros sintomas, após período de uso continuado de voz ${ }^{32}$. Muitos indivíduos passam a valorizar a voz após o uso profissional ou, até mesmo, após alguma experiência com problemas de saúde vocais. Assim, na atuação do teleatendimento o fonoaudiólogo pode contribuir para a saúde do atendente/operador, além de colaborar com resultados desejados pela empresa identificando falhas na comunicação e propiciando seu aprimoramento ${ }^{33}$. Diversos trabalhos brasileiros têm abordado a importância das ações voltadas aos profissionais da voz com vistas à prevenção de alterações vocais em função das limitações sociais e profissionais que podem provocar na vida desses sujeitos ${ }^{34}$. Um estudo com voz profissional em um grupo de atores verificou a importância de realizar avaliações objetivas para verificar o tipo de trabalho a ser usado afim de, redirecionar o treinamento vocal, mostrando o benefício desta prática no direcionamento do treino e na compreensão de como os hábitos pessoais e abusos vocais influenciam na produção da voz ${ }^{35}$.

Nos relatos dos consultores, referentes à avaliação individual da participação nas oficinas, foram possíveis observar manifestações positivas de todos os indivíduos, ou seja, as oficinas foram consideradas importantes e produtivas. Um curso de formação com teleatendentes sobre voz propicia bons resultados em relação aos cuidados com a voz, conforme descrito em alguns relatos:

"Achei super produtivo, pois fazia um monte de coisas erradas que melhorei"; "Percebi o quanto é importante cuidar da voz, e que simples exercícios fazem grande diferença"; "Achei muito positivo. Principalmente por fazer com que eu parasse para pensar sobre a saúde da minha voz"; "Gostei do fato de terem nos esclarecido a importância de cuidarmos da nossa voz e como fazê-lo".

Identificou-se a importância das oficinas para os consultores, bem como as diversas atividades trabalhadas. Além disso, relataram sobre o tempo de duração e da dificuldade de horários que tinham, já que todos ou estavam em atendimento, ou saindo para outras atividades não relacionadas ao VIVAVOZ.
"Aprendi que é importante proteger a saúde das nossas pregas vocais."; "Os exercícios que mais gostei foram os de vibrar"; "Gostei do exercício do som nasal, de aquecimento e desaquecimento vocal"; "Achei legal a parte da respiração"; "Achei poucos encontros para desenvolver o hábito de fazer os exercícios todos os dias"; "Alguns horários não pude fazer em razão da faculdade".

Embora, os relatos tenham destacado os benefícios de fazer as oficinas, dificuldades também foram citadas. Estas são importantes para reflexão e organização das futuras ações fonoaudiológicas com os teleoperadores do call center VIVAVOZ. Além da percepção e do envolvimento dos mesmos com as atividades de voz, os consultores fizeram sugestões:

"Os hábitos incorretos são mais difíceis de corrigir"; "Para mim é difícil transformá-los em um hábito diário"; "Acho interessante haver oficinas de continuidade"; "Sugiro que as oficinas sejam mais frequentes, porque é muito legal e importante."

Dentre as limitações do estudo, destaca-se o número reduzido da amostra e a falta de adesão dos consultores às oficinas. Uma vez que trabalhadores em situação de tensão ou estresse podem achar positivo qualquer tipo de atenção dada a eles salienta-se que no presente estudo essas variáveis não foram controladas o que pode ter influenciado no relato dos consultores.

\section{CONCLUSÃO}

Na presente pesquisa, realizada com os teleoperadores do VIVAVOZ, observaram-se mudanças positivas na qualidade do atendimento à população, além da ampliação do conhecimento dos teleatendentes em relação ao uso correto da voz e manutenção do bem-estar vocal. Ainda, existem questões a serem respondidas, como por exemplo, o tempo considerado saudável para estes operadores e a possibilidade de um aquecimento de voz diário, apropriado para atenuar a fadiga vocal. Ao compreender esses aspectos, pode-se aproximar um pouco mais da realidade desses profissionais, bem como de suas necessidades vocais.

\section{AGRADECIMENTOS}

SENAD/AAPEFATO oferecem o apoio financeiro para o funcionamento e para as pesquisas desenvolvidas no VIVAVOZ, além da bolsa de doutorado (T.C.M). Reconhece-se também o apoio do CNPq, através de bolsa de produtividade de pesquisa 1C (H.M.T.B.). Agradecimento aos consultores VIVAVOZ. 


\begin{abstract}
Purpose: to determine effects of a program of vocal well-being for consultants of a call center for health. Methods: the study involved 27 consultants of the Call Center VivaVoz who were assessed before and after voice training by analysis of perceptual voice quality, articulation, speech rate, resonance, intensity and frequency through the utterance of sustained vowels and connected speech with counting from 1 to 20 and the issue of the week. Voice recording was done in the digital system and the Japanese scale GRBAS-I as an analytical tool. The study also included self-assessment survey of vocal and vocal symptoms. Activities voice were divided into five workshops on vocal health, techniques for heating and cooling, articulation and breathing. Descriptive and bivariate analysis were performed using t test for paired samples and McNemar test. Results: pre-intervention results are that $50 \%$ of women and $33 \%$ of male consultants showed changes related to breathing, $50 \%$ of consultants with changes in vocal quality and $33 \%$ of men difficulties with the joint. After the workshops, the results showed improved voice quality, standard vocalization and fluency of speech and voice satisfaction reports from consultants. Conclusion: there was a positive change in quality of care to the population, in addition to increasing the knowledge on telemarketing staff for the correct use of voice and maintaining the well-being vocal.
\end{abstract}

KEYWORDS: Voice; Consultants; Voice Training

\section{REFERÊNCIAS}

1. Silva MCB, Cunha MB, Souza CCL, Mitre El. Avaliação do processamento auditivo em operadores de telemarketing. Rev. CEFAC, Oct./ Dec. 2006; 8(4): 536-42.

2. Moreira T, Beraldin G, Cielo C, Trinca M. Hábitos vocais dos operadores de telemarketing. J Bras Fonoaudiol. 2005; 5(22):331-5.

3. Pedroso, Maria Ignez de Lima.O uso de técnicas vocais como recursos retóricos na construção do discurso. Revista do GEL. 2008,5 (2):139-61.

4. Azevedo LL, Vianello L, Oliveira HGP, Oliveira IA, Oliveira BFV, Silva CM. Queixas vocais e grau de disfonia em professoras do ensino fundamental. Rev. soc. bras. fonoaudiol. 2009 ;14(2): 192-6.

5. Oliveira, A.G.A., Behlau, M., Gouveia, N. Vocal Symptoms in Telemarketers: A Random and Controlled Field Trial Folia Phoniatr Logop 2009;61:76-82

6. Penteado RZ, Pereira IMTB. Qualidade de vida e saúde vocal de professores. Rev. Saúde Pública. 2007; 41(2): 236-43.

7. Ferreira AEM, Silva VOS. Preservação da voz é forte tendência em recursos humanos. UNIVOZ. [homepage na internet] São Paulo: 2002. [Acesso em 23/12/2009] Disponível em: URL: http://www. univoz.com.br/shop/shop.dll/noticias28d7.html

8. Silveira DN, Brasolotto AG. Reabilitação vocal em pacientes com doença de Parkinson: fatores interferentes. Pró-Fono. 2005; 17(2):241-50.

9. Ferreira LP, Costa H. Voz ativa: falando sobre o profissional da voz. São Paulo: Roca; 2000.
10. Barros HM, Santos V, Mazoni C, Dantas DC, Ferigolo M. Neuroscience education for health profession undergraduates in a call-center for drug abuse prevention. Drug Alcohol Depend. 2008 Dec; 98(3):270-4.

11. Mazoni CG, Bisch NK, Freese L. Aconselhamento telefônico reativo para cessação do consumo do tabaco: relato de caso. Aletheia. 2006; 24:137-48.

12. Costa MFL, Barreto SM. Tipos de estudos epidemiológicos: conceitos básicos e aplicações na área do envelhecimento. Epidemiol. Serv. Saúde [periódico na Internet]. Dez. 2003 [acesso em 21 fev 2010]; 12 (4): 1-13. Disponível em: http://scielo. iec.pa.gov.br/pdf/ess/v12n4/v12n4a03.pdf

13. Bacha SMC, Ribeiro JL, Camargo AFP. Ação fonoaudiológica em Central de Telemarketing In: Ferreira LP, Andrada SMA. Saúde vocal práticas fonoaudiológicas. 1. ed. São Paulo: Roca; 2002. p. 309-52.

14. Ferreira AEM, Fujita RR. Avaliação do Profissional da Voz. Vox Brasilis [periódico na Internet]. Abril 2002 [acesso em 21 fev 2010]; 8 (2): 10-11. Disponível em: http://www.ablv.com.br/vox/VOX\% 202\%20-\%20pdf.pdf

15. Hirano M. Clinical examination of voice. New York: Springer-Verlag; 1981.

16. Behlau M, Madazio G, Feijó D, Pontes P. Avaliação da voz. In: Behlau M. Voz: o livro do especialista. vol 1. Rio de Janeiro: Revinter; 2008.

17. Behlau M, Pontes P. Higiene vocal. Rio de Janeiro: Revinter; 1999.

18. Saenz-Lechon N, Godino-Llorente JI, OsmaRuiz V, Blanco-Velasco M, Cruz-Roldan F. 
Automatic assessment of voice quality according to the GRBAS scale. Conf Proc IEEE Eng Med Biol Soc. 2006; 1:2478-81.

19. Ferreira LP, Akutsu CM, Luciano P, Viviano NAG. Condições de produção vocal de teleoperadores: correlação entre questões de saúde, hábitos e sintomas vocais. Rev Soc Bras Fonoaudiol. 2008; 13(4):307-15.

20. Penteado, RZ. Relação entre saúde e trabalho docente: percepção de professores sobre saúde vocal. Rev Soc Bras Fonoaudiol. 2007; 12:18-22.

21. Salzstein RBW, Costa HO. Operador de telemarketing: relações entre trabalho $\mathrm{e}$ queixas vocais. In: Alloza RG, Salzstein RBW. Fonoaudiologia na empresa: atuação em call center. Rio de Janeiro: Revinter; 2002, p 55 -82.

22. Penteado RZ, Pereira IMTB. A voz do professor: relações entre trabalho, saúde e qualidade de vida Rev. Bras. Saúde Ocup 1999; 25(95/96):109-30. 23. Jones K, Sigmon J, Hock L, Nelson E, Sullivan $\mathrm{M}$, Ogren F. Prevalence and risk factors for voice problems among telemarketers. Arch Otolaryngol Head Neck Surg [periódico na internet]. Maio 2002 [acesso em 21 março 2010]; 128: 571-77. Disponível em: http://archotol.highwire.org/cgi/ reprint/128/5/571.

24. Ueda KH, dos Santos LZ, Oliveira IB. 25 anos de cuidados com a voz profissional: avaliando ações. Rev CEFAC. 2008; 10(4):557-65.

25. Ferreira LP, Eliane BC, Ana CBA, Marta AAS. Assessoria fonoaudiológica: análise de um processo de construção entre o fonoaudiólogo e o teleoperador. Dist Comun Hum. 2008; 20(2):219-28. 26. Lehto L, Rantala L, Vilkman E, Alku P, Bäckström T. Experiences of a Short Vocal Training Course for Call-Centre Customer Service Advisors. Folia Phoniatr Logop [ periódico na internet]. Julho 2003 [acesso em 21 março 2010]; 55:163-176. Disponível em: http://lib.tkk.fi/Diss/2007/isbn9789512286980/ article1.pdf.
27. Farghaly SM, Andrade CRF. Programa de treinamento vocal para locutores de rádio. Rev Soc Bras Fonoaudiol. 2008; 13(4):316-24.

28. Behlau M, Madazio G, Feijó D, Azevedo R, Gielow I, Rehder MI. Aperfeiçoamento vocal e tratamento fonoaudiológico das disfonias. In: Behlau M. Voz: o livro do especialista. Vol 2. Rio de Janeiro: Revinter; 2005. p. 409-564.

29. Hazlett DE, Duffy OM, Moorhead SA. Occupational voice demands and their impact on the call-centre industry. BMC Public Health. 2009; 9:108.

30. Santos IR. Possibilidades de assessoria fonoaudiológica em centrais de telemarketing. [monografia]. Rio de Janeiro (RJ): CEFAC Pós-graduação em Saúde e Educação; 2003.

31. Algodoal MJA. Voz profissional: o operador de telemarketing. [tese/mestrado]. São Paulo (SP): Pontifícia Universidade Católica - Mestrado em distúrbios da comunicação; 1995.

32. Vintturi J, Alku P, Lauri ER, Sala E, Sihvo M, Vilkman I. Objective analysis of vocal warm-up with special reference to ergonomic factors. J Voice. Março, 2001; 15(1):36-53.

33. Garcia RAS. Operadores de uma central de telemarketing: os múltiplos sentidos da voz. [tese/ mestrado]. São Paulo (SP): Pontifícia Universidade Católica- Mestrado em Linguagem; 2000.

34. Regina Y. S. Chun, Emilse A. M. Servilha, Luciana M. A. Santos, Maísa H. Sanches. Promoção da Saúde: o conhecimento do aluno de jornalismo sobre sua voz - Distúrb Comun, São Paulo, 2007;19(1):73-80.

35. Harvey PL, Feudo PJ, Aronson DB. Objective analysis of actors'voices: an initial report. J.Voice. Junho, 1989; 3(2): 143-7.

DOI: 10.1590/S1516-18462010005000109

RECEBIDO EM: 10/11/2009

ACEITO EM: 23/05/2010

Endereço para correspondência:

VIVAVOZ

Taís de Campos Moreira

Rua Sarmento Leite, $2453^{\circ}$ andar

Porto Alegre - RS

E-mails: taiscm@ufcspa.edu.br taiscmoreira@ hotmail.com 